\title{
Avaliação psicológica: importância e domínio de atividades segundo docentes
}

\section{Psychological assessment: importance and knowledge of activities according to professors}

\author{
Ana Paula Porto Noronha* \\ Universidade São Francisco - USF, I tatiba, São Paulo, Brasil \\ Mariana Varandas de Camargo Barros** \\ Universidade São Francisco - USF, Itatiba, São Paulo, Brasil \\ Maiana Farias Oliveira Nunes*** \\ Faculdade Avantis, Florianópolis, Santa Catarina, Brasil \\ Acácia Aparecida Angeli dos Santos**** \\ Universidade São Francisco - USF, I tatiba, São Paulo, Brasil
}

\begin{abstract}
RESUMO
O presente estudo visou investigar, por meio do Questionário de Competência para Avaliação Psicológica - QCAP, a percepção de importância e de domínio de algumas competências em avaliação psicológica. Participaram 98 docentes de disciplinas variadas dos cursos de formação para a graduação em psicologia. Os itens considerados mais importantes foram 'aplicar os princípios éticos' e 'saber comunicar os resultados'. Já os menos importantes foram 'noções de estatística' e 'conhecer uma ampla gama de testes'. Pôde-se constatar que os escores médios dos docentes que ministram disciplinas referentes à avaliação psicológica foram significativamente superiores aos daqueles que ministram outras disciplinas, tanto no que se refere à importância como ao domínio apresentado nos quesitos medidos pelo questionário.
\end{abstract}

Palavras-chave: formação profissional, teste psicológico, avaliação de docentes.

\footnotetext{
ABSTRACT

The present study aimed at investigate the importance and competence perception for some psychological assessment skills with a questionnaire named "Questionário de Competência para Avaliação Psicológica - QCAP". 98 professors from various disciplines in Psychology courses took part on this study. The items considered most important were "applying ethical principles" and "knowing how to communicate test results". However, the least important ones were "having basic statistical knowledge" and "knowing a wide range of tests". Professors of psychological assessment disciplines had significantly higher means both at items reflecting the given importance
} 
and also for competence perception, when compared to professors of other disciplines not related to psychological assessment.

Keywords: professional education, psychological test, teacher's evaluation.

\section{I ntrodução}

É inerente à postura do profissional de psicologia a preocupação com o bem-estar de toda pessoa que esteja sob sua responsabilidade, quer como profissional, quer como pesquisador. Sob essa perspectiva, Anastasi (1972) apontou alguns princípios que devem ser encontrados em um bom profissional da área de avaliação psicológica. Entre eles, pode-se destacar a responsabilidade como cientista, professor e profissional, o reconhecimento dos limites de sua competência, o respeito aos padrões morais e legais, a garantia da segurança dos testes realizados pelos examinandos, a confiança nas editoras e as precauções com as pesquisas. Tais asserções são ainda debatidas nos âmbitos nacional e estrangeiro (Hoff, 1999; Iliescu, Ispas, \& Harris, 2011; Urbina, 2007, dentre outros). Adicionalmente, Primi (2010) acrescenta que um segmento particular da avaliação psicológica compreende a criação de instrumentos e técnicas.

Mais especialmente, a American Psychological Association (2000) destacou que, independentemente do contexto em que realiza avaliação, o Psicólogo deve ter como função essencial fazer interpretações válidas dos escores dos testes, coletadas por meio de fontes variadas, realizando uma adequada seleção de instrumentos, respeitando procedimentos de aplicação e de correção. É conferida uma grande importância à capacidade do profissional de saber integrar as informações coletadas, conhecer o construto avaliado, manter-se atualizado, além de ter clareza dos objetivos da testagem e do público alvo da mesma. Adicionalmente, também é explicitada a relevância do psicólogo praticar suas funções sob supervisão de um profissional com experiência apropriada.

No que se refere ao uso de instrumentos, Anastasi e Urbina (2000) valorizam a aprovação de leis estaduais de licenciamento e certificação para os psicólogos, nas quais cada habilitação caracterizar-se-ia por competências e habilidades específicas, sendo que o licenciamento controlaria a prática dos profissionais. No entanto, ainda não há sistema com objetivo semelhante para os profissionais que atuam com avaliação psicológica no país. Apesar da resolução do Conselho Federal de Psicologia (CFP $n^{\circ}$ 002/2003) explicitar claramente as condições para o uso e a construção de testes psicológicos, um Psicólogo pode se utilizar de quaisquer tipos de testes, independentemente de seu nível de complexidade e de ter formação especializada para tanto. 
A formação em avaliação psicológica no Brasil há décadas atrás foi apontada como deficiente por alguns autores. A título de exemplo, Pereira e Carellos (1995) destacaram a deficiência do ensino e transmissão das teorias, técnicas e práticas dos exames psicológicos. Segundo as autoras, para um bom ensino, o Professor deveria fazer com que seu aprendiz conhecesse a fundamentação teórica subjacente ao teste psicológico, desenvolvesse habilidades relativas à aplicação, correção e análise dos resultados dos testes e tivesse acesso a uma prática efetiva e supervisionada. Com isso, as autoras esperam que seja possível a formação de um profissional competente, que utilize técnicas de forma adequada e com embasamento teórico consistente. Adicionalmente, elas destacam que seria desejável que os Psicólogos da área assumissem postura ética e "intranquilidade científica", o que contribuiria para o desenvolvimento da área.

Em direção semelhante, Bock (2005) destaca alguns problemas do ensino de avaliação psicológica, como o ensino de técnicas afastadas da realidade e da ética, a falta de empenho em pesquisas, a relação autoritária do Professor-aluno e o ensino de 'como' aplicar alguns instrumentos, e não de 'porque' aplicá-los. A este respeito, o Conselho Federal de Psicologia - CFP (2004), por meio da Resolução CFP no 08/2004, institui que o planejamento acadêmico nos cursos de psicologia deve assegurar a adequada aplicação e avaliação de estratégias, técnicas, recursos e instrumentos psicológicos. Já Gomes (2000) enfatiza a necessidade de se mudar a postura desinteressada e descrente em relação ao uso dos testes. Para tanto, esta transformação implicaria em um esforço conjunto de Professores, pesquisadores, profissionais, conselhos de classe e demais órgãos comprometidos com a preparação de psicólogos.

Na mesma perspectiva, Paula, Pereira e Nascimento (2007) advertem que a Avaliação Psicológica faz parte das especificidades profissionais do Psicólogo, porém, além de dominar as técnicas, é necessário destreza de apreensão e integração dos dados obtidos. As autoras apresentam um estudo sobre a formação na área de Avaliação Psicológica realizado com 358 estudantes de Psicologia que cursavam o último ano da Graduação de universidades particulares de Minas Gerais. As autoras verificaram que os alunos consideram a formação em Avaliação Psicológica insuficiente, e observaram que muitos deles não conheciam, apesar de estar no final do curso, a Resolução CFP no 02/2003 do CFP, que regulamenta o uso e comercialização de testes psicológicos.

É importante ressaltar que nos últimos anos no Brasil houve um maior interesse dos Psicólogos pela Avaliação Psicológica, que pode ser notado pelo aumento do número de congressos específicos da área; criação de laboratórios ligados à universidade; aumento de linhas de pesquisa sobre o tema em programas de pós-graduação, 
bem como a criação de curso de mestrado e doutorado específicos na área (Hutz \& Bandeira, 2003; Noronha \& Repold, 2010). Outras autoras apontaram que, apesar do crescimento, é urgente a necessidade de incremento da formação dos psicólogos em avaliação psicológica (Noronha \& Freitas, 2005), inclusive aproximando o campo das reflexões sobre o atendimento aos princípios éticos e à garantia dos direitos humanos (Oliveira, Nuernberg, \& Nunes, 2013). Para o desenvolvimento do conhecimento científico na área de Avaliação Psicológica, Noronha et al. (2002) afirmam que teoria da medida e psicometria, avaliação da inteligência, avaliação da personalidade e elaboração de laudos devem ser conteúdos imprescindíveis nos cursos de Psicologia. Outros aspectos foram ressaltados por Silva (2004) e por Noronha e Freitas (2005), como a importância do domínio de teorias e o uso adequado de instrumentos de coleta de dados, tais como testes e técnicas. As autoras realçam a importância de sustentar tal necessidade com a Psicometria, definida como uma abordagem científica, que trata das teorias, das técnicas, dos instrumentos (testes, escalas, inventários, questionário) e dos diferentes modelos estatísticos e/ou matemáticos de avaliação e mensuração de atributos subjetivos. A ciência da medida é a responsável por fazer com que as avaliações sejam fidedignas, válidas, padronizadas e livres de vieses. Essa posição é congruente com algumas referências estrangeiras, dentre as quais, Almeida (1999) e Anastasi e Urbina (2000), entre outras.

Quanto ao uso dos testes, a pesquisa feita por Noronha (2002) com 214 psicólogos inscritos no CRP/6a Região buscou identificar os problemas mais graves e frequentes no uso de testes psicológicos. A autora detectou, à época, que os psicólogos apontaram que os instrumentos representam o mais grave problema para a atuação em Avaliação Psicológica (32,9\%), visto que mencionaram dificuldades relacionadas à sua construção, às suas características psicométricas, às revisões e às normas. Com relação à frequência dos problemas, novamente foram referidos os instrumentos $(37,8 \%)$ e, em acréscimo, a formação profissional $(27,5 \%)$.

Resultados semelhantes aos dos estudos brasileiros foram obtidos por Diniz, Almeida e Pais (2007) quando buscaram caracterizar os Psicólogos portugueses e suas práticas na área de Avaliação Psicológica. No total, 265 Psicólogos portugueses em exercício de profissão há, no mínimo, três anos, responderam a um questionário. Entre os resultados, pôde-se observar que a Psicologia Clínica $(64,3 \%)$ é o contexto mais presente e $78,9 \%$ representa o sexo feminino. No total, $20,5 \%$ dos profissionais cursaram uma especialização, $23,6 \%$ são mestres, 9,5\% são doutores e $16 \%$ estão cursando algum tipo de pós-graduação. A Avaliação Psicológica apareceu como atuação principal pelos profissionais. Os participantes indicaram que haveria necessidade de mais investimento nos 
seguintes aspectos para a melhor utilização dos testes, a saber, a melhoria da formação, a validação de testes em Portugal, a supervisão da prática e a maior divulgação dos testes.

No que se refere à formação dos docentes de Avaliação Psicológica no Brasil, Noronha (2003) investigou 75 professores de Psicologia. A autora encontrou que eles possuíam, em sua maioria, título de Especialização $(38,7 \%)$ e Mestrado (35,3\%). Discutiu-se que, embora os docentes tenham experiência profissional, sua baixa titulação ainda é um fator que deve ser avaliado com seriedade. A autora afirma que a competência docente apropriada deveria ser capaz de prover aulas consistentes e uma formação sólida para os alunos; e por fim, a pesquisa deveria ser urgentemente vinculada à prática profissional.

Em uma pesquisa com 172 Professores de disciplinas de Técnicas de Exame Psicológico (TEP), provenientes de 13 Estados do Brasil, Alves, Alchieri e Marques (2002) buscaram mensurar a quantidade de testes psicológicos ensinados nos cursos de Graduação, de acordo com os Professores. Os autores destacaram que, embora os entrevistados apontassem 10 testes como sendo um número mínimo para ser lecionado em um curso de psicologia, em média são ensinados 14,46 testes, ou seja, em termos de quantidade, são lecionados mais testes ao longo do curso que o considerado mínimo. Os autores salientam que a constante atualização dos programas de ensino deve proporcionar a maximização do potencial dos futuros profissionais de Psicologia, assim como a multiplicação de uma educação mais efetiva e eficiente.

Mais recentemente, Noronha e Reppold (2010) fizeram uma reflexão crítica sobre a avaliação psicológica atual e a que se deseja ter no contexto brasileiro. As autoras expressaram grande preocupação com a formação na área. Duas considerações finais foram destacadas por elas, quais sejam: competência e segurança nas práticas avaliativas devem ser exigidas dos Psicólogos que fazem uso da avaliação; e formação continuada deve ser entendida como imprescindível ao profissional de Psicologia.

Tendo em vista as questões apontadas a respeito da preparação profissional do Psicólogo e, em particular, as que se relacionam com a Avaliação Psicológica, o presente estudo visou investigar a concepção de Psicólogos Docentes de variadas disciplinas do curso de Psicologia sobre a importância de atividades referentes à prática de avaliação, bem como o domínio que acham que possuem delas. Além disso, comparou-se os resultados considerando as variáveis tempo de formado, região do país, idade, titulação de professores e o exercício da docência em áreas diversas. O presente estudo diferencia-se dos já mencionados por possibilitar uma comparação dos aspectos que Professores de disciplinas de Avaliação Psicológica e de outras áreas consideram mais relevantes para esta prática, assim como a 
competência percebida em si no que diz respeito aos mesmos aspectos.

\section{Método}

\section{Participantes}

A presente pesquisa foi realizada com 98 Professores universitários, sendo $78,6 \% \quad(n=77)$ do sexo feminino e $21,4 \% \quad(n=21)$ do masculino, com média de 38,9 anos de idade $(D P=9,76)$ e 15,5 anos de formação $(D P=8,93)$. Com relação à formação, $66,3 \% \quad(n=65)$ dos participantes eram especialistas, $66,3 \%(n=65)$ tinham título de Mestre e $20,4 \% \quad(n=20)$, o de Doutor. Os participantes eram residentes de doze estados brasileiros, sendo que o Nordeste foi representado com $21,4 \%(n=21)$, o Centro Oeste, com $5,1 \%(n=5)$, o Sudeste, com $69,4 \%(n=68)$ e o Sul com $4,1 \%(n=4)$.

Com relação à realização de Avaliação Psicológica, 63,3\% do total de participantes disseram realizá-la em diversos contextos, sendo citados 20, obtendo destaque a Clínica $(21,3 \%)$ e a Seleção de Pessoal/Organizacional $(11,5 \%)$. Sobre a utilização de testes psicológicos, $59,2 \%$ do total de participantes disseram fazer uso dele no seu cotidiano profissional.

Foi feita uma análise das disciplinas lecionadas pelos docentes em parte da amostra $(\mathrm{N}=49)$. Verificou-se que havia 68 títulos de disciplina distintos, sendo que as mais frequentes foram: 'Estágio(s) Supervisionado(s)' $(8,1 \%)$, seguido do 'Trabalho de Conclusão de Curso - TCC' $(6,8 \%)$ e da 'Psicanálise' I, II, III e IV (6,1\%).

Para fins de análise, foram considerados Professores de Avaliação Psicológica aqueles que lecionam disciplinas como 'Introdução à Avaliação Psicológica', 'Avaliação Psicológica Aplicada', 'Conceitos de Psicometria', 'Técnicas de Exame Psicológico', 'Medidas em Psicologia' e 'Ensino de Testes'. Houve 11 títulos relacionados à Avaliação Psicológica, dentre eles, 'Avaliação Psicológica' (5,0\%), 'Técnica de Exame e Avaliação Psicológica - TEAP' (4,4\%), 'Psicodiagnóstico' $(3,7 \%)$, 'Técnica de Exame Psicológico - TEP' (2,5\%), 'Psicometria' $(1,2 \%)$, 'Técnicas Projetivas' (1,2\%), 'Testes Cognitivos e Motores' $(0,6 \%)$, 'Técnicas de Entrevista e Observação' $(0,6 \%)$, 'Inventário de Personalidade e Técnicas Gráficas' $(0,6 \%)$, 'Testes e Exames Psicológicos' $(0,6 \%)$ e 'Testes Objetivos' $(0,6 \%)$, representando apenas $21 \%$ de todas as disciplinas. Dos participantes que ministram disciplinas relacionadas à Avaliação Psicológica, 57,9\% possuem Especialização, 68,4\% são Mestres e $31,6 \%$ fizeram, ou estão fazendo,o Doutorado. Nem todos os participantes que lecionam disciplinas de Avaliação Psicológica utilizam-na em seu exercício 
profissional (15,8\%, não realizam avaliação) e nem utilizam testes (5,3\% não utilizam testes).

\section{Material}

Foi aplicado o Questionário de Competências para Avaliação Psicológica - QCAP (Noronha, Nunes, \& Ambiel, 2007), que é dividido em três partes. A primeira solicitava informações sobre a identificação do participante e a segunda, sobre a formação profissional. A terceira consistia em um questionário de 20 questões organizadas em escala Likert de três pontos (de 1 a 3), sobre a importância que é dada às atividades profissionais relacionadas à Avaliação Psicológica e o domínio percebido sobre elas. O instrumento foi elaborado com base nas definições do Test User Qualification da American Psychological Association (APA, 2000) e englobava o conhecimento psicométrico e de medidas em avaliação, a seleção de instrumentos apropriados, os procedimentos de administração dos testes, aspectos éticos e a experiência supervisionada, entre outros.

Procedimentos de coleta de dados

Após a aprovação do projeto por um Comitê de Ética em Pesquisa, a sistemática de aplicação deu-se de duas formas distintas. Um grupo de participantes foi abordado em um congresso nacional de psicologia, realizado no ano de 2006 (49 participantes) e outra parte da amostra foi convidada para a participação na pesquisa pela rede de contatos pessoais da autora (49 participantes). Ambas as aplicações duraram em média, 15 minutos. O Termo de Consentimento Livre e Esclarecido foi assinado por todos que responderam ao questionário.

\section{Resultados}

Os dados foram analisados quantitativamente, buscando descrever as percepções de domínio e importância relacionados à avaliação psicológica. $\mathrm{Na}$ análise geral dos itens do questionário, os participantes tiveram como média de importância 55,3 $(D P=7,04)$ e $46,5(\mathrm{DP}=10,41)$ para o domínio. Vale destacar que a pontuação mínima e máxima possível é de 20 e 60 pontos, respectivamente. Esses resultados indicam que, embora esses psicólogos julguem que há um rol considerável de atividades importantes, o domínio delas não está sempre presente. Já no que se refere à análise descritiva dos itens do instrumento, as questões foram analisadas individualmente e algumas apresentaram um resultado que vale ser destacado. Com vistas a verificar se as diferenças eram significativas, 
realizou-se o teste não paramétrico Related-Samples Friedman's, cujo coeficiente foi altamente significativo $(p<0,000)$.

Quanto à importância atribuída pelos profissionais aos aspectos referidos, destacaram-se como mais importantes os itens 'Aplicar os Princípios Éticos', 'Saber Comunicar Resultados', 'Conhecer os Aspectos Legais' e 'Condições Adequadas de Testagem'. Aspectos considerados menos importantes foram 'Noções de Estatística', 'Conhecer Ampla Gama de Testes' e 'Conhecer Conceitos de Psicometria'.

No que se refere às questões que avaliavam o domínio pessoal na concepção dos profissionais, destacaram-se como os mais frequentes 'Aplicar os Princípios Éticos', 'Saber Comunicar Resultados', 'Conhecer os Aspectos Legais' e 'Condições Adequadas de Testagem'. Como os menos frequentes estiveram presentes 'Noções de Estatística', 'Conhecer Conceitos de Psicometria' e 'Conhecer Ampla Gama de Testes'. De igual modo, o teste não paramétrico Related-Samples Friedman's foi utilizado, e o resultado indicou coeficiente altamente significativo $(p<0,000)$.

Com relação a elaborar parecer psicológico, 91,8\% dos Docentes disseram ser muito importante, porém, apenas 59,2\% admitem ter alto domínio. Com relação ao conhecimento dos conceitos de psicometria, $64,3 \%$ acharam muito importante e, a maioria, $56,1 \%$, reconhece ter domínio médio. Já o conhecimento dos construtos psicológicos foi avaliado por $89,8 \%$ dos participantes como muito importante embora somente $48 \%$ indicaram ter alto domínio. Por fim, 96,9\% consideraram muito relevante dominar a aplicação dos princípios éticos, mas apenas 76,5\% percebem-se com alto domínio.

Realizou-se uma correlação entre as questões relativas à importância e ao domínio cujos resultados estão ilustrados na Tabela 1. Os coeficientes de correlação significativos estão apresentados em negrito nessa tabela. Para melhor compreensão da Tabela, convém esclarecer que a questão '01_d' representa o domínio dos princípios éticos, enquanto a questão ' 01 i' refere-se à importância dada ao mesmo aspecto, e assim sucessivamente. 
Tabela 1. Coeficientes de correlação entre as questões de Importância (I) e Domínio (D).

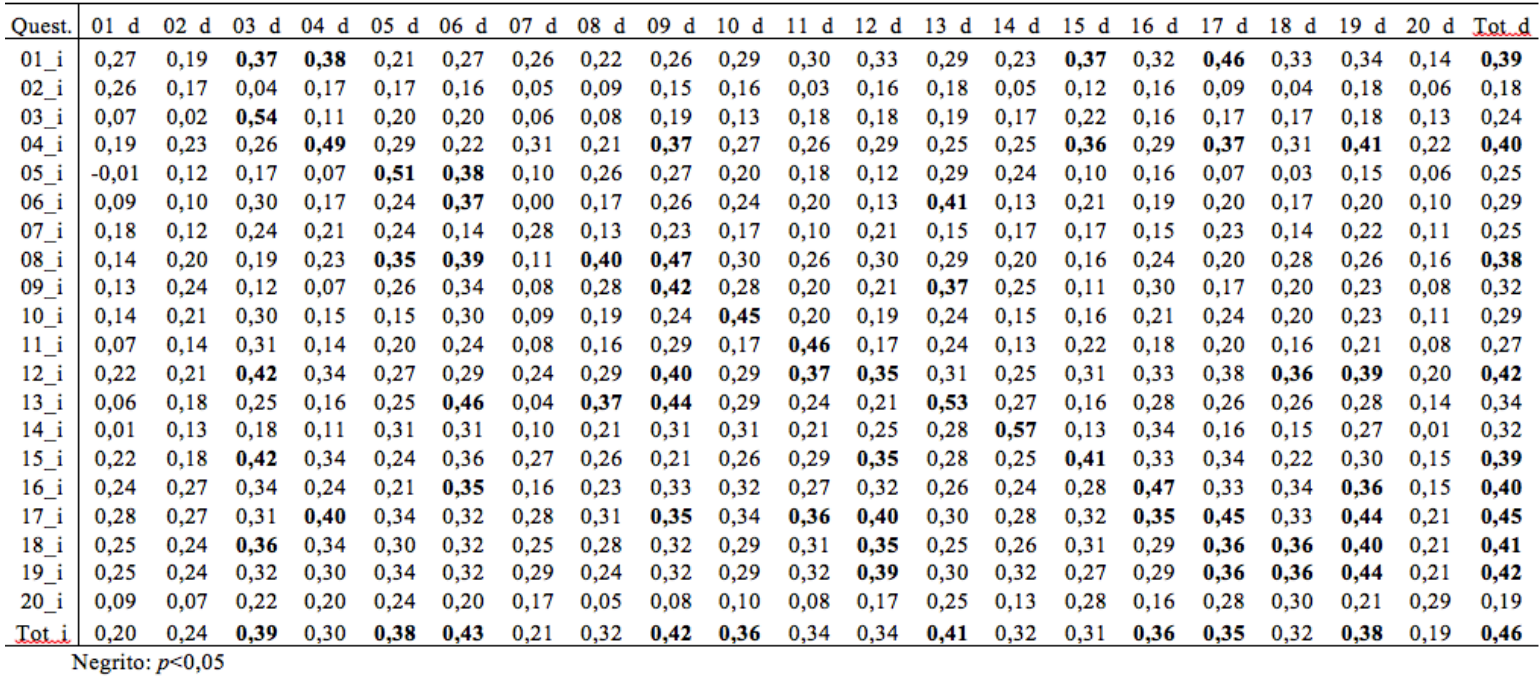

Esperava-se que muitos dos coeficientes de correlação obtidos na linha diagonal da tabela fossem significativos, uma vez que, nesse caso, ele indica a magnitude da correlação entre a importância atribuída a um dado quesito e a percepção que o respondente tem sobre o seu domínio. Pode-se perceber que quatro índices de correlação não foram significativas na linha diagonal, sendo elas referentes a 'Aplicação dos Princípios Éticos', 'Compreender a Fundamentação Teórica', 'Elaboração de Parecer Psicológico' e 'Ter Experiência Supervisionada'. Os coeficientes de correlação significativa da linha diagonal variaram de 0,35 a 0,57.

Em 57 casos, houve correlações moderadas (acima de 0,35) fora da linha diagonal. Alguns deles são ilustrativos de aspectos que não se pressupunha necessariamente a existência de correlações significativas. Como exemplo pode-se citar a questão de domínio, 'Compreender a Psicopatologia' (3_d) que apresentou correlação positiva e significativa com a importância dada a 'Aplicação dos Princípios Éticos' (1_i). Entre outras, também a questão de importância de ter 'Noções de Estatística' (13_i) apareceu correlacionada positiva e significativamente à importância dada a 'Saber Comunicar Resultados' (17_i).

Ainda com relação aos coeficientes apresentados na Tabela 1, a questão 'Selecionar instrumentos para as situações de avaliação psicológica' (questão 12) teve correlações positivas fora da linha diagonal, tanto para a importância, quanto para o domínio. Houve correlação do domínio desta questão com os seguintes itens de importância: 'Reconhecer a Natureza dos Problemas' (15_i), 'Saber Comunicar Resultados' (17_i), 'Conhecer os Aspectos Legais' (18_i) e 'Conhecer Condições Adequadas de Testagem' (19_i). Com relação à mesma questão, houve correlações significativas fora da linha diagonal entre a importância e as seguintes questões de domínio: 
'Compreender Psicopatologia' (quest.3_d), 'Entender os Estudos de Validade e Precisão' (9_d), 'Conhecer os Aspectos Legais' (18_d) e 'Conhecer Condições Adequadas de Testagem' (19_d).

Outra análise foi resultante da comparação entre o tempo de formado dos professores e as diferentes percepções de domínio e importância. Para tanto, reuniu-se os participantes segundo a informação que forneceram sobre o tempo de formado, ficando com três grupos. 0 primeiro deles composto por Docentes com até 10 anos de formado $(n=33)$, o segundo, entre 11 e 20 anos de formado $(n=34)$ e o terceiro de 21 a 30 anos $(n=29)$, sendo que dois sujeitos não disponibilizaram tal informação. Pôde-se perceber que os participantes formados há menos tempo não tiveram médias significativamente diferentes daqueles formados há mais tempo tanto em relação à importância como no domínio percebido, considerando os escores gerais.

Ainda com relação aos grupos com diferentes períodos de formado, realizou-se a análise de variância (ANOVA) para os itens separadamente e percebeu-se que houve diferença marginalmente significativa para a importância de 'Conhecer a Psicopatologia' $[F(2,93)=2,975 ; p=0,056]$ e 'Noções de Estatística' $[F(2,93)=2,755$; $p=0,069]$, e significativa para o domínio da 'Elaboração de Parecer Psicológico' $[F(2,93)=3,418 ; p=0,037]$ e 'Entender os Estudos de Validade e Precisão' $[F(2,93)=4,269 ; p=0,017]$. Por meio da prova post-hoc de Tukey, verificou-se que os formados há mais tempo (entre 21 e 30 anos) tiveram média significativamente menor que os formados entre 11 e 20 anos. Já quanto ao entendimento dos estudos de validade e precisão, os formados há mais tempo (entre 21 e 30 anos) novamente tiveram média significativamente menor que os formados entre 1 e 10 anos.

No que concerne às faixas etárias, os participantes foram categorizados em três grupos, sendo o primeiro até 33 anos $(27,6 \%)$, o segundo de 34 até $44(31,6 \%)$ e o terceiro de 45 até $59(24,5 \%)$, destacando que $16,3 \%$ não disponibilizaram tal informação. Houve diferenças significativas em duas questões após a aplicação da ANOVA, sendo que para a importância destacou-se a 'Interpretação de Tabelas de Manuais' $[F(2,79)=5,096 ; p=0,008]$, 'Noções de Estatística' $[F(2,79)=5,778 ; p=0,005]$. Já para o domínio, os itens que apresentaram diferença de média significativa foram 'Conhecer os Construtos Psicológicos' $[F(2,79)=3,872 ; p=0,025]$ e 'Noções de Estatística' $[F(2,79)=3,304 ; p=0,590]$.

Dadas as diferenças encontradas entre as faixas etárias e visando a identificação de quais grupos se diferenciavam recorreu-se à prova post-hoc de Tukey, realizadas tanto para as questões de domínio, quanto às de importância que apresentaram diferença significativa associada à idade. Apenas uma das questões 'Conhecer os Construtos Psicológico' teve o aumento de pontuação progressivo em razão da 
ordem cronológica, possivelmente representando o reconhecimento da importância e do domínio sendo gradativo conforme o aumento da idade.

A fim de verificar a existência de diferenças referentes à região de residência dos Docentes também se aplicou a análise de variância. Os resultados obtidos permitiram inferir sobre a inexistência de diferenças significativas, quer para o total de importância $[F(3,94)=0,915 ; \quad p=0,437]$ ou para o total do domínio $[F(3,94)=0,441 ; p=0,725]$.

No que diz respeito a diferenças relacionadas à titulação dos participantes, encontrou-se diferenças significativas para a importância percebida de 'Conhecer a Psicopatologia' $[F(3,94)=2,682 ; \quad p=0,051]$, 'Conhecer Conceitos de Psicometria' $[F(3,94)=3,193 ; p=0,029]$ e 'Entender os Estudos de Validade e Precisão' $[F(3,94)=3,193 ; p=0,027]$. Ao realizar a prova de Tukey observou-se que as médias não acompanharam o nível hierárquico pressuposto de competência dos Docentes, a saber: 'graduado', 'especialista', 'Mestre' e 'Doutor'. Os resultados da ANOVA para a percepção de domínio, envolvendo as diferentes titulações não evidenciou nenhuma diferença significativa, sendo 0 total $[F(3,94)=0,182 ; p=0,909]$.

No que se refere à comparação dos participantes quanto às disciplinas lecionadas, foram organizados três grupos, quais sejam, os que ministram apenas disciplinas ligadas à Avaliação Psicológica $(16,3 \%)$, os que lecionam outras disciplinas que não envolvem Avaliação Psicológica $(59,2 \%)$ e os que além de ministrarem disciplinas de Avaliação Psicológica, também ministram outras de áreas diferentes $(24,5 \%)$.

Os resultados da ANOVA revelaram ausência de diferença estatisticamente significativa para importância $[F(2,46)=1,429$; $p=0,236]$ e diferença significativa apenas para $o$ domínio $[F(2,49)=6,658 ; p=0,003]$. A prova de Tukey revelou que os que ministram outras disciplinas e os que lecionam Avaliação Psicológica se diferenciam em dois conjuntos distintos, sendo que o de Avaliação Psicológica, como era esperado, obteve média mais elevada.

\section{Discussão}

Dada a relevância do ensino da avaliação psicológica, que é uma das únicas atividades profissionais de atribuição exclusiva do Psicólogo, o estudo aqui apresentado debruçou-se sobre a percepção da importância e do domínio de professores universitários quanto a atividades relativas ao tema. Os dados obtidos mostram aspectos interessantes sobre a questão pesquisada.

Inicialmente, com a caracterização da amostra, pode-se perceber que 
pouco mais da metade dos participantes cursou uma Especialização ou um Mestrado e que menos de um quarto dos docentes fez o Doutorado. Quando separados os participantes que ministram disciplinas relacionadas à Avaliação Psicológica, o percentual de titulados Stricto Sensu (Mestres e Doutores) aumenta. Esta realidade confirma os achados de Noronha (2003) e de Diniz, Almeida e Pais (2007). Mais especialmente, apontam a necessidade de se acompanhar até que ponto a titulação dos Docentes contribui com a melhoria da formação dos alunos.

Ainda no que se refere a características da amostra, o contexto clínico foi um dos mais citados pelos Docentes, quando o assunto foi a aplicação da Avaliação Psicológica. Resultado similar foi obtido na pesquisa de Diniz, Almeida e Pais (2007), o que revela a predominância desse contexto na atuação do Psicólogo: temática amplamente discutida em cenário nacional, no que se refere à ampla concentração de Psicólogos naquele contexto.

No que concerne à análise geral das médias de importância e de domínio, verificou-se que o domínio das atividades não corresponde à importância atribuída, aparentando um possível despreparo profissional, o que já foi evidenciado por autores, dentre os quais, Noronha e Reppold (2010) e Pereira e Carellos (1995). Os índices de correlação dos itens de domínio e importância correspondentes não foram tão altos e quatro deles não foram significativos, sendo os referentes a 'Compreender a Fundamentação Teórica', 'Aplicação dos Princípios Éticos', 'Elaboração de Parecer Psicológico' e 'Ter Experiência Supervisionada'. Vale destacar que estas são questões de grande importância para a atuação profissional, levando a pensar que falta investimento na capacitação dos psicólogos, tal como preconizado por Noronha et al. (2002). Esses tópicos talvez possam se tornar foco de investimento na formação dos psicólogos, uma vez que se notou um descompasso entre a importância atribuída e o domínio percebido, o que pode ser objeto de investigação de futuros estudos. Pode-se hipotetizar que os Docentes considerem os aspectos éticos importantes, porém ao considerar a prática profissional, achem que os patamares éticos estabelecidos são muito difíceis de serem concretizados no seu dia a dia.

A correlação mais alta fora da diagonal $(r=0,47)$ foi entre a percepção de domínio referente ao item 'Entender os Estudos de Validade e Precisão' e a importância de 'Conhecer a Construção de um Instrumento de Medida', ou seja, duas atividades que são complementares. Já a correlação mais alta dentro da diagonal ( $r=$ $0,57)$, ou seja, referente ao domínio e à importância conferidos ao mesmo item, foi com relação à 'Usar e Interpretar Instrumentos', o que pode ser identificado como coerência da análise do respondente. Considerando o tempo de formado, pôde-se perceber que os participantes, cuja titulação foi obtida mais recentemente não tiveram 
médias significativamente diferentes tanto em relação à importância, quanto ao domínio, que aqueles titulados há mais tempo, considerando os escores gerais. Ainda no quesito da formação, foi possível verificar que não houve diferença entre os Docentes no que tange à percepção de domínio. Em relação aos dados, esperava-se que cursos de Pós-Graduação (Stricto ou Lato sensu) promovessem a formação continuada e maior competência técnica percebida em profissionais mais titulados (Alves, Alchieri, \& Marques, 2002). Por certo, tal informação, aliada às considerações, no sentido de que parece ser necessário avaliar se a titulação dos Docentes contribui com preparação mais adequada dos alunos, devem ser consideradas. O presente estudo não atenderá o escopo dessa problematização, mas deve-se considerar em estudos futuros se a formação Stricto Sensu tem atendido o objetivo de formar Docentes para Ensino Superior, além de pesquisadores.

Outros estudos desta natureza devem ser desenvolvidos, abrangendo um maior número de Docentes e detalhando a investigação sobre a relação entre o curso de Pós-Graduação escolhido e a percepção de domínio associada. Considera-se como limitação do estudo, o número de Docentes consultados, bem como a falta de composição de uma amostra diversificada. Por fim, um dado que deve ser mais bem investigado é o porquê de haver poucas diferenças significativas no domínio percebido de acordo com o tempo de formado. Espera-se que esta e outras pesquisas possam contribuir para a formulação de políticas que visem à superação das carências da formação profissional e despertar nos Docentes o desejo de aperfeiçoamento.

\section{Referências}

Almeida, L. S.(1999). Avaliação Psicológica - exigências e desenvolvimento nos seus métodos. In:S. M. Wechler \& R.S.L. Guzzo(Orgs.). Avaliação Psicológica: perspectiva internacional (PP 41-55). São Paulo: Casa do Psicólogo.

Alves, I. C. B., Alchieri, J. C. \& Marques, K. C.(2002). As técnicas de exame psicológico ensinadas nos cursos de graduação de acordo com os professores. Psico-USF, 7(1), 77-88.

American Psychological Association (2000). Report of the task force on test user qualifications. Washington: DC: Autor.

Anastasi, A.(1972). Campos da psicologia aplicada. São Paulo: Herder.

Anastasi, A.\& Urbina, S.(2000). Testagem psicológica. 7. ed. Porto Alegre: Artmed.

Bock, A. M. B.(2005). Novo projeto para profissão no Brasil: contribuições para a formação. Recuperado em janeiro, 2008, de http://www. abepsi.org.br/web/artigos.aspx. 
Conselho Federal de Psicologia (CFP) (2003). Resolução no. 02/2003. Recuperado em 02 maio, 2006, de http://www.pol.org.br.

Conselho Federal de Psicologia (CFP) (2006). Resolução no. 08/2004. Recuperado em 02 maio, 2006, de http://www.pol.org.br.

Diniz, A. M., Almeida, L. S.\& Pais, L. G. (2007). Contextos profissionais e práticas da Avaliação Psicológica: inquérito aos psicólogos portugueses. Psico-USF, 12(1), 1-12.

Iliescu, D., Ispas, D.\& Harris, M.(2011). Social implications and ethics of testing. Recuperado em 07 novembro, 2011, de http://www.intestcom.org/Publications/ORTA/Social\% 20implica tions $\% 20$ and $\% 20$ ethics $\% 20$ of\% 20testing.php.

Gomes, I. C.(2000). A formação em psicodiagnóstico e testes psicológicos. Psicologia: Teoria e Prática, 2(2), 60-69.

Hoff, M. S.(1999). A proposta de diretrizes curriculares para os cursos de psicologia: uma perspectiva de avanços? Psicologia: Ciência e Profissão, 19 (3), 12-31.

Hutz, C. S.\& Bandeira, D. R.(2003). Avaliação psicológica no Brasil: situação atual e desafios para o futuro. In: O.H. Yamamoto \& V.V. Gouveia (Eds.). Construindo a Psicologia Brasileira: desafios da ciência e prática psicológica. (PP. 621-277). São Paulo: Casa do Psicólogo.

Noronha, A. P. P.(2002). Os problemas mais graves e mais freqüentes no uso dos testes psicológicos. Psicologia: Reflexão e Crítica, 15(1), 135-142.

Noronha, A. P. P.(2003). Docentes de Psicologia: formação profissional. Estudos de Psicologia , 8(1), 169-173.

Noronha, A. P. P., Nunes, M. F. O, Ambiel, R. A. M. (2007). Questionário de Competências para Avaliação Psicológica QCAP. São Paulo: Universidade São Francisco. Não publicado.

Noronha, A. P. P. \& Freitas, F. A.(2005). Testes psicológicos, usos e conhecimento. Psico,36(1), 21-28.

Noronha, A. P. P., Ziviani, C., Hutz, C. S., Bandeira, D. R., Custódio, E. M., Alves, I. C. B. et al. (2002). Em Defesa da Avaliação Psicológica. Avaliação Psicológica, 2(2),173-174.

Noronha, A. P. P. \& Reppold, C. T.(2010). Considerações sobre a avaliação psicológica no Brasil. Psicologia: Ciência e Profissão, 30 (número especial), 192-201.

Oliveira, C. M., Nuernberg, A. H.\& Nunes, C. H. S. S. (2013). Desenho universal e avaliação psicológica na perspectiva dos direitos humanos. Avaliação Psicológica, 12( 3), 421-428.

Paula, A. V., Pereira, A. S.\& Nascimento, E.(2007). Opinião de alunos de psicologia sobre 0 ensino em Avaliação Psicológica. PsicoUSF, 12(1), 33-43.

Pereira, A. P. C. \& Carellos, S. D. M. S.(1995). Examinando o ensino das Técnicas de Exame Psicológico. Caderno de Psicologia, 3(4), 33-36. 
Primi, R.(2010). Avaliação psicológica no Brasil: fundamentos, situação atual e direções para o futuro. Psicologia: Teoria e Pesquisa, 26 (n. esp.), 25-35.

Silva, J. A.(2004). O uso da avaliação e a avaliação de seu uso. Paidéia, 14(29), 255-264.

Urbina, S. (2007). Fundamentos da testagem psicológica. Porto Alegre: Artmed.

\section{Endereço para correspondência \\ Ana Paula Porto Noronha}

Universidade São Francisco

Programa de Pós Graduação Stricto Sensu em Psicologia

Rua Alexandre Rodrigues Barbosa, 45, Centro, CEP 13251-900, Itatiba - SP, Brasil

Endereço eletrônico: ana.noronha@usf.edu.br

\section{Mariana Varandas de Camargo Barros}

Universidade São Francisco

Programa de Pós Graduação Stricto Sensu em Psicologia

Rua Alexandre Rodrigues Barbosa, 45, Centro, CEP 13251-900, Itatiba - SP, Brasil

Endereço eletrônico: mariana.barros.psi@gmail.com

\section{Maiana Farias Oliveira Nunes}

Faculdade Avantis

Curso de Psicologia

Av. Marginal Leste, 3600, KM 132, Bairro dos Estados, CEP 88339-125, Balneário Camboriú - SC, Brasil

Endereço eletrônico: maiananunes@mac.com

\section{Acácia Aparecida Angeli dos Santos}

Universidade São Francisco

Programa de Pós-Graduação Stricto Sensu em Psicologia

Rua Alexandre Rodrigues Barbosa, 45, Centro, CEP 13251-900, Itatiba - SP, Brasil

Endereço eletrônico: acacia.santos@usf.edu.br

Recebido em: 23/03/2012

Reformulado em: 14/04/2014

Aceito para publicação em: 09/06/2014

\section{Notas}

* Doutora em Psicologia Ciência e Profissão, pela Pontifícia Universidade Católica de Campinas. Docente do Programa de Pós-Graduação Stricto Sensu em Psicologia da Universidade São Francisco. Bolsista Produtividade em Pesquisa do CNPq.

** Mestre em Psicologia pelo Programa Pós-Graduação Stricto Sensu em Psicologia da Universidade São Francisco.

*** Doutora em Psicologia pelo Programa de Pós-Graduação Stricto Sensu em Psicologia da Universidade São Francisco. Docente do curso de Psicologia da Faculdade Avantis.

**** Doutora em Psicologia, pela Universidade de São Paulo. Docente do Programa de Pós Graduação Stricto Sensu em Psicologia da Universidade São Francisco. Bolsista Produtividade em Pesquisa do CNPq. 